\title{
Direct Measurement of Lateral Correlations under Controlled Nanoconfinement
}

\author{
P. Kékicheff, ${ }^{1,2, *}$ J. Iss, ${ }^{1}$ P. Fontaine, ${ }^{2}$ and A. Johner ${ }^{1}$ \\ ${ }^{1}$ Institut Charles Sadron, Université de Strasbourg, CNRS UPR22, 23 rue du Loess 67034 Strasbourg cedex 2, France \\ ${ }^{2}$ Synchrotron SOLEIL, Saint Aubin, 91192 Gif-sur-Yvette, France
}

(Received 7 July 2017; published 15 March 2018)

\begin{abstract}
Lateral correlations along hydrophobic surfaces whose separation can be varied continuously are measured by x-ray scattering using a modified surface force apparatus coupled with synchrotron radiation, named SFAX. A weak isotropic diffuse scattering along the equatorial plane is revealed for mica surfaces rendered hydrophobic and charge neutral by immersion in cationic surfactant solutions at low concentrations. The peak corresponds to a lateral surface correlation length $\xi \approx 12 \mathrm{~nm}$, without long-range order. These findings are compatible with the atomic force microscopy imaging of a single surface, where adsorbed surfactant stripes appear surrounded by bare mica zones. Remarkably, the scattering patterns remain stable for gap widths $D$ larger than the lateral period but change in intensity and shape (to a lesser extent) as soon as $D<\xi$. This evolution codes for a redistribution of counterions (counterion release from antagonistic patches) and the associated new x-ray labeling of the patterns. The redistribution of counterions is also the key mechanism to the long-range electrostatic attraction between similar, overall charge-neutral walls, reported earlier.
\end{abstract}

DOI: 10.1103/PhysRevLett.120.118001

Controlled (e.g., patchy) or random inhomogeneously charged layers have recently attracted much attention again [1]. The disorder in the surface charge can be quenched [2] or annealed [3]. While the interaction between surfaces in the annealed case mainly involves polarization effects somewhat similar to van der Waals interactions, the quenched case is very sensitive to counterion release [4]. Some theoretical efforts were devoted to quenched systems involving multivalent counterions [5] for which strong and unexpected effects were described [6].

Even the interaction between homogeneously charged surfaces in water with monovalent counterions, for which the standard Poisson-Boltzmann mean-field theory [7] is naively expected to hold, turns out much more complicated due to the subtle interplay of the familiar electrostatics and counterion entropy with the structure of liquid water close to the surface [8-10]. (Although some insight into the chemistry-dependent, local dielectric response was gained recently from chemically realistic molecular dynamics simulations combined with the analytical theory [11].) Actually, even the sign of this interaction seems impossible to get from a generic continuous theory at short separations, and a microscopic description taking water explicitly into account is needed [9]. Luckily, at separations surpassing one (or a few) nanometer(s), the classical PoissonBoltzmann theory turns out to work surprisingly well [9]. Accurate measurements sometimes reveal moderate corrections to the mean field [12].

A simple and standard way to control (modify) the charge of a surface is to incubate it in a solution of oppositely charged adsorbing species, typically adsorbing charged surfactants [13]. One of us studied mica surfaces with a bare area per (negative) elementary charge $1 / \sigma_{0}=$ $48 \AA^{2}$ incubated in cationic surfactant (hexadecyltrimethylammonium bromide, CTAB) solutions of increasing concentration [14]. The surface force apparatus experiment measures repulsion at both very low and high CTAB concentrations [14]. In a narrow concentration range well below the critical micellar concentration of CTAB in water, the interaction is found attractive. The attraction was attributed to charge fluctuations which prevail over the weak average charge in the narrow regime of charge inversion $[15,16]$. This suggests some favorable correlation between the charge fluctuations on both surfaces. A direct measurement of surfactant-surfactant correlations by an in situ scattering technique is called for. The idea to set up such a technique came later [17,18], but the realization of an accurate apparatus faced major challenges.

It is generally accepted that charged systems can adopt (more or less regular) microstructures rather than undergo macroscopic phase separation at no or low salt, the so-called compatibility enhancement by charge [19]. Typically, short-range repulsion between the incompatible species drives the separation, and the local electric neutrality breaking or the entropy of the counterions oppose charge separation. Microphase separation then appears as a compromise. The incomplete $\mathrm{CTA}^{+}$monolayer on the oppositely charged mica is somewhat similar. Separation between a regular dense layer and the bare mica would separate the opposite charges or confine the counterions clouds of anions (cations) over the corresponding areas of 
$\mathrm{CTA}^{+}$layers (bare mica). On the other hand, macroscopic separation optimizes the contact between water and the hydrophobic tails and reduces the associated line tension (2D surface tension) contribution. A microstructured system like a layer with an array of holes or stripes alternating $\mathrm{CTA}^{+}$layers and bare mica could be a compromise. In the case of charge compensation between the mica and adsorbed $\mathrm{CTA}^{+}$considered here, a paradigmic purely electrostatic system without salt (and counterions) is instructive. Take a striped structure where the mica has charge density $-\sigma$ (possibly $\sigma_{0}$ ) and the covered area occupies twice as large stripes of (net) charge $\sigma / 2$. (This peculiar choice is motivated by experimental observations described below.) The cell of the periodic system is a stripe of width $L$ comprising one central bare substripe of width $L / 3$ flanked symmetrically by two covered substripes of width $L / 3$. The electrostatic energy density per unit area is calculated straightforwardly as $E_{\text {el }}=\left[13 /\left(4 \pi^{2}\right)\right] \zeta(3) \times$ $L l_{B} \sigma^{2} k_{B} T$, with $\zeta($.$) the Riemann zeta function [\zeta(3) \approx$ $1.20]$ and $l_{B}$ the Bjerrum length; in water, $l_{B}=0.7 \mathrm{~nm}$. On the other hand, the period of width $L$ contains two layer edge lines which correspond to the free energy density $2 \gamma / L$. There obviously is an optimal period given by $\left(L_{0}^{*}\right)^{2}=\left\{8 \pi^{2} /[13 \zeta(3)]\right\}\left[\left(\gamma / k_{B} T\right) /\left(l_{B} \sigma^{2}\right)\right]$. As anticipated, the period increases with the line tension and decreases with the strength of the electrostatic interaction. If we assume the surface charge $-\sigma_{0}$ for the bare mica and the line tension $\gamma \approx 10 k_{B} T / \mathrm{nm}$, we arrive at a small period $L_{0}^{*} \approx 2.5 \mathrm{~nm}$. Beyond this purely electrostatic case, counterions (and salt) diminish the electrostatic interaction and hence increase the period $L^{*}$. Next we try to include all effects on a phenomenological basis.

We expect each substripe to qualitatively retain its counterions, similarly to a macroscopic homogeneous stripe, provided it is wider than the Gouy-Chapman length $\lambda$, recalled below, within which the counterions are qualitatively confined. Indeed, from the expression $E_{\mathrm{el}}$, the energy per charge $\sim L / \lambda$ increases linearly with the stripe width and exceeds the thermal energy for $L \gtrsim \lambda$. Given the complexity of the system and the impact of local interactions, a phenomenological approach seems appropriate. We hence split the excess free energy density into a contribution of long-range interactions and a contribution of all kind of local interactions, lumped into the line tension. For a homogeneous layer with a net charge density $\sigma$, say, negative, and monovalent counterions, the PoissonBoltzmann mean-field theory does apply, and in the absence of salt the electrostatic potential $\psi(z)$ grows logarithmically with the distance $z$ from the wall: $\psi=2 k_{B} T \log [(z+\lambda) / \lambda]$ with $1 / \lambda=2 \pi l_{B}|\sigma|$. The GouyChapman contribution to the free energy surface density of the layer is obtained through a charging process as $F_{\mathrm{GC}}=k_{B} T \int_{0}^{\sigma}\left[\psi_{s}\left(\sigma^{\prime}\right)-\psi_{\infty}\left(\sigma^{\prime}\right)\right] d \sigma^{\prime}$, where the square brackets give the potential difference between the surface and infinity in thermal units. The reference state is taken without surface charge and counterions. This energy is physically well defined, and in practice the convergence of the integral is ensured by some strong cutoff of the electrostatic potential away from the surface. The ever-present Debye-Hückel cutoff due to traces of salt $\left(\mathrm{CTA}^{+} ; \mathrm{Br}^{-}\right.$; dissociation of water) ensures a cutoff at the Debye length $\kappa^{-1}$. Provided the cutoff length is much larger than the (nanometric) Gouy-Chapman length, then the free energy contribution $F_{\mathrm{GC}} \approx 2 k_{B} T \sigma \log \left(\kappa^{-1} / \lambda\right)$. Let us now take, for the sake of simplicity, a symmetric periodic stripe structure of stripe width $L / 2$ and alternating surface charge density $\pm \sigma$. When seen from a distance (much) larger than $L$, the surface appears globally neutral and structureless. Assuming low salt and a Debye length much larger than $L$, the cutoff length beyond which the potential is almost $\psi_{\infty}$ is now defined by $L$. Assuming further that $L \gg \lambda$, the free energy of the striped layer per unit area reads $F_{\text {stripe }} \approx$ $2 k_{B} T \sigma \log (L / \lambda)$, where we should subtract the GouyChapman free energy density of the bare mica layer which is independent of $L$. Let now $\gamma$ be the line tension at the stripe borders, its contribution to the free energy density is $2 \gamma / L$. The minimization of the sum of Gouy-Chapman free energy density and line tension energy of the striped structure defines the period $L^{*} \approx\left[\gamma /\left(k_{B} T \sigma\right)\right]$. In summary,

$$
L_{0}^{*}=\sqrt{\frac{8 \pi^{2}}{13 \zeta(3)} \frac{\gamma / k_{B} T}{l_{B} \sigma^{2}}} ; \quad L^{*} \approx \frac{\gamma}{k_{B} T \sigma} .
$$

The period $L^{*}$ carries an extra factor $\sqrt{\gamma l_{B} / k_{B} T}$ with respect to $L_{0}^{*}$, for the previously retained values of the parameters $\sqrt{\gamma l_{B} / k_{B} T} \approx 2.6$, and $L^{*}$ falls in the range $\sim 10 \mathrm{~nm}$. The crossover at $\gamma l_{B} / k_{B} T \sim 1$ corresponds to a period $L^{*} \sim L_{0}^{*} \sim \lambda$. In practice, the structure is correlated over a number $n_{\xi}$ of periods which confers the width $\sim 2 \pi /\left(n_{\xi} L\right)$ to the "Bragg peak." Coming back to the case of interacting surfaces, the structure, if any, should be stable at least down to separations $\sim L^{*}$. If the mobility of the system allows, structures on opposite plates can correlate at smaller separation (with bare patches preferentially facing covered ones), and, eventually, the structure could be deeply modified at the closest distances. We rather expect that arrangements of bare and covered areas are too slow, but counterions could respond. The pattern is then quenched but counterions redistribute; the main effect is counterion release when antagonistic (bare and covered) patches face accompanied by a counterion increase between agonistic patches. As counterions dominate the scattering, their (local) release or increase merely amounts to a new $\mathrm{x}$-ray labeling of the pattern with the contrast (almost stepwise) increasing upon the approach of the surfaces. In the quenched case, the dominant effect is an almost homogeneous variation of the scattered intensity with a superimposed minor deformation and almost no displacement of the scattering peak. Note that the same counterion 
release mechanism is the main source of the attraction between the two surfaces $[1,15]$.

To get experimental insight, we purposely designed a surface force apparatus (SFA) coupled with small angle $\mathrm{x}$-ray scattering. This advanced device with enhanced spatial and time resolution and automation, named SFAX (Fig. 1), was specifically built for the SIRIUS beam line [20] at the synchrotron radiation facility SOLEIL, France. Here it provides in situ structural information on the in-plane organization of the layers confined between two macroscopic (mica) surfaces or in their vicinity. The experiment was carried out for separations ranging from tens of micrometers down to molecular contact using the absolute distance scale measurement, the atomic resolution, and the crossed-cylinder geometry on which the surface force apparatus technique is based [22,23]. These two hemicylinders are hollow with centered small rectangular apertures (4 $\mathrm{mm}$ long along the summit ridge axis; $1.5 \mathrm{~mm}$ wide) and are mounted with their axes crossed at right angles. The separation between the two surfaces could be varied (from tens of micrometers down to mechanical contact) with a piezo element superimposed on the coarse positioning range of a motorized micropositioner. The surface separation was measured by interferometry in white

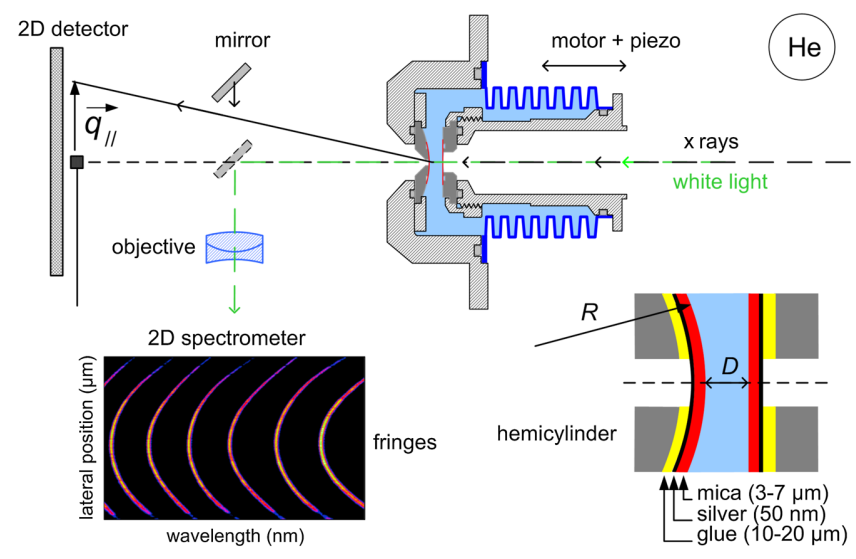

FIG. 1. Schematic of the experiment set on the SIRIUS beam line [20] at the synchrotron radiation facility SOLEIL. The incident $\mathrm{x}$ rays fall at normal incidence on the confining mica surfaces of the SFAX, allowing the equatorial plane of the reciprocal space to be described. Back-silvered atomically smooth thin mica sheets $(\approx 5 \mu \mathrm{m}$ thick) are glued down on hollow hemicylinders. Interferometry in white light allows the surface separation to be determined at $\pm 0.2 \mathrm{~nm}$. The upstream mica surface can be moved to approach or separate from the fixed exit mica surface. Taking advantage of the surface position stability (drift $<1 \mathrm{~nm} / \mathrm{min}$ [21]), the mirrors and objectives used to collect the light and the interferometric pattern are moved aside the optical axis. The SFAX is placed in an enclosure, from the beryllium window at the direct beam exit to the $2 \mathrm{D}$ detector at a $\sim 4.5 \mathrm{~m}$ distance from the SFAX, filled with helium to avoid spurious scattering by air and also oxidation of the $50 \mathrm{~nm}$ thick silver layer coating the back of the mica sheets and its degradation under high incident flux of $\mathrm{x}$ rays. light as in the classical SFA technique [24] (with a sensitivity of $0.2 \mathrm{~nm}$ [21]). At every separation gap set between the two surfaces, the scattering pattern was measured by irradiating the confined sample at $8 \mathrm{keV}$. In this transmission geometry, the incident $\mathrm{x}$-ray beam impinges normally to the two confining mica surfaces (Fig. 1). The scattering vectors $Q$ are parallel to the mica surfaces, and the equatorial plane of the reciprocal space is described. By placing the 2D detector (PILATUS3, Dectris Ltd., Switzerland) at about $4.5 \mathrm{~m}$ distance from the fixed confining mica surface at the exit of the SFAX, the confined structure was investigated at small angle scattering ( $Q$ range, $5 \times 10^{-3}-0.12 \AA^{-1}$ ). With a flux more than $10^{10}$ photons $/ \mathrm{sec} / \mu \mathrm{m}^{2} / 100 \mathrm{~mA}$ and a divergence of less than $0.1 \mathrm{mrad}$, good statistics and a satisfactory signal to noise ratio were obtained with exposure times ranging from $10 \mathrm{~min}$ to $2 \mathrm{~h}$. The surface separation was always checked before and after scattering record. Thanks to the very good stability of our homebuilt SFAX, in terms of thermal and mechanical drift of the surfaces, the surface separation can be considered as not changing much during the collection of each scattering pattern $(<5 \%)$.

A weak diffuse scattering ring whose scattering vectors are parallel to the surfactant-coated mica surfaces is revealed in the equatorial plane (Fig. 2). When the surface separation $D$ is large enough, the magnitude and the position at $Q_{\|}^{*} \approx 0.054 \AA^{-1}$ of this diffuse ring almost do not evolve as well as the overall scattering pattern (Fig. 3). Conversely, the intensity and the shape (to a lesser extent)

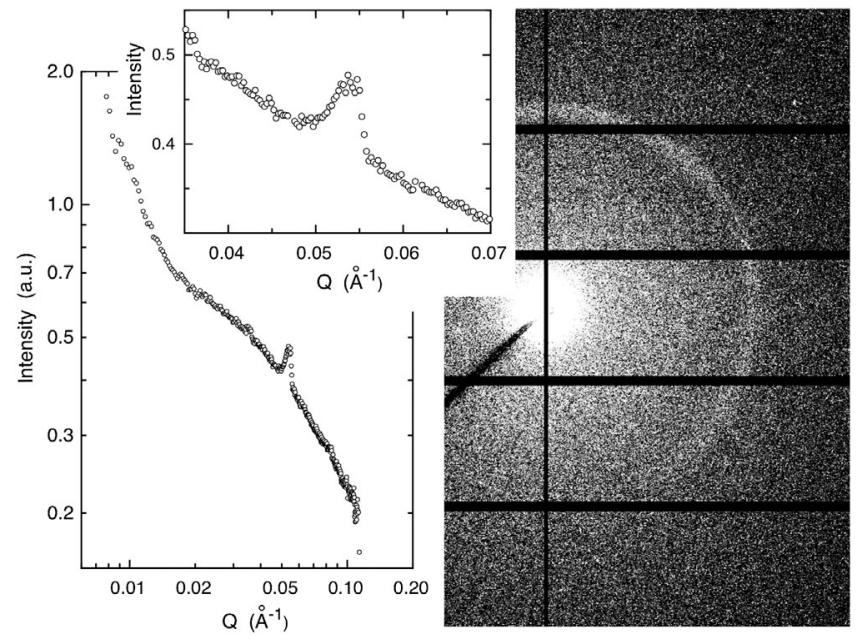

FIG. 2. A slice at constant intensity of the scattering pattern observed in the geometry of Fig. 1 for mica surfaces immersed in an aqueous $\mathrm{CTAB}$ solution at $\approx 1 / 120 \mathrm{cmc}$ ( $24 \mathrm{~h}$ equilibration) and separated by $\sim 100 \mathrm{~nm}$. The beamstop, placed in the middle and mounted on a rod, houses a pin diode detector to monitor the sample transmission. After the background correction and normalization of the detector efficiency, an isotropic diffuse ring shows up at small angles in the equatorial plane when the slice is made at low intensity levels. Left: Radial averaging scattering pattern, with an enlargement of the peak. 
of the latter are affected for $D \leq \xi^{*}=2 \pi /\left(Q_{\|}^{*}\right) \approx 12 \mathrm{~nm}$. These evolutions suggest a subtle interplay between the characteristic length set by the microstructure and the gap width - the correlation length $\xi$ along the planes and the separation $D$ along the normal direction-with a remarkable crossover occurring at $D \sim \xi^{*}$. Figure 3 illustrates the evolution of the diffuse scattering passing from large to small separations in the confinement gap. An almost stepwise increase of the intensity at separation $\xi^{*}$ by about $80 \%$ is followed by a smoother evolution. The position of the diffuse scattering bump observed in the equatorial plane suggests a lateral correlation length $\xi^{*} \approx 12 \mathrm{~nm}$ along the hydrophobic substrate. The absence of second order underlines that there is no order propagation at long range; its width would correspond to a domain size (correlation length) of $\approx 50 \mathrm{~nm}$. The fact that both the position and the shape of the diffuse bump are not greatly affected when the crossover $D \sim \xi^{*}$ is passed in either direction (behavior fully reversible) suggests a slight or slow structural rearrangement of the adsorbed hydrophobic layers, provided they have not come into contact. Indeed, a mechanical contact of the two opposite CTAB-coated surfaces induces cavitation [14] and irreversible structural changes (data not

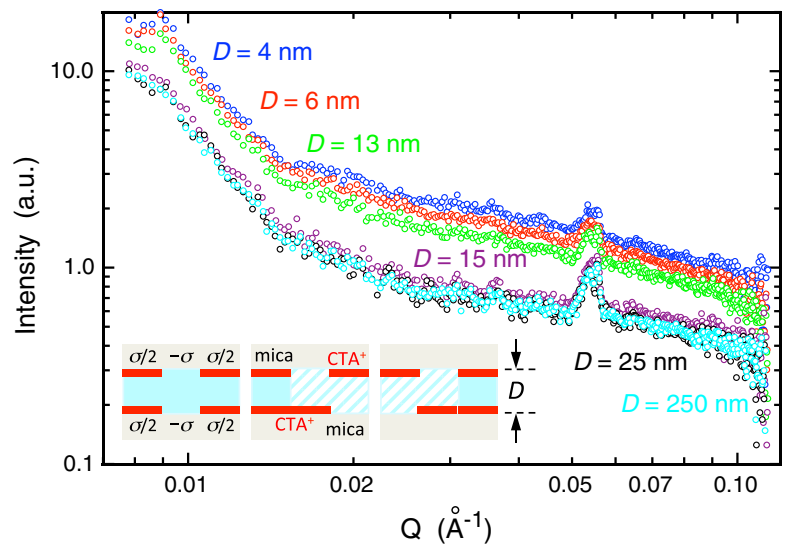

FIG. 3. Scattering curves (corrected for the scattering contribution of the mica sheets immersed in pure water using standard subtraction procedures) for different separations $D$ between the CTAB coated-mica surfaces. The scattering is independent of $D$ at high separations and nearly independent at low ones, with a stepwise (almost uniform) increase between $D=15$ and $13 \mathrm{~nm}$. This is merely attributed to the redistribution of counterions ( $\mathrm{Br}^{-}$from $\mathrm{CTAB}$ and $\mathrm{K}^{+}$dissociated from mica) when the structures on opposing surfaces start to interact. Antagonistic patches release their excess counterions, which leave as neutral salt, while agonistic patches take up some more counterions. The latter effect is weaker. With a period composed of three substripes, the inset illustrates three simple situations with the very same parallel structure but shifted by $1 / 3$ period either way. In the left case, the bare mica and monolayer both attract excess counterions; the two other cases facing monolayers attract more $\mathrm{Br}^{-}$, and antagonistic regions (hatchings) release the $\mathrm{Br}^{-}$and half of the $K^{+}$. In all cases, the contrast is enhanced and the scattering of the period increased at the transition. reported). The (almost stepwise) intensity enhancement of the overall diffuse scattering as soon as the spatial crossover is attained matches our expectation for quenched patterns. As discussed above, the dominating mechanism is a local counterion release or increase.

The microstructuration of the adsorbed surfactant layer is also indisputably evidenced by AFM imaging single mica surfaces that were incubated under exactly the same solution conditions (Fig. 4) under the recent peak force tapping mode allowing substrates to be imaged with the lowest possible applied force [25]. In the height mode, bare mica regions left without any adsorption are clearly revealed (although one cannot exclude the presence of isolated CTAB molecules lying flat). They are surrounded by an adsorbed film covering $\approx 65 \%$ of the area interpreted as a hydrophobic monolayer, since its thickness is close to the value previously measured by direct force measurement in the same solution conditions [14]. The images look similar to those from the simulation of 2D mixtures [26] [see Fig. 1(a) therein] and are compatible with the scattering diagram obtained from the SFAX (Fig. 2) when their Fourier Transforms are calculated (Fig. 4). The revealed dominating length of about $15 \mathrm{~nm}$ is (slightly) larger but of the same order as the correlation length evidenced by the scattering SFA experiment $(\approx 12 \mathrm{~nm})$, the discrepancy being due to the image convolution with the tip radius (3-10 nm) of similar order. Indeed, the nonlinear process of dilation of the sample and erosion of the dilated [27] image induces a shift of the peak to lower values [28]. Its width, about 5 times larger than from $x$ rays, reflects the ratio of the linear sample sizes. The overall shapes of the FTs are similar but at small $Q$ (due to
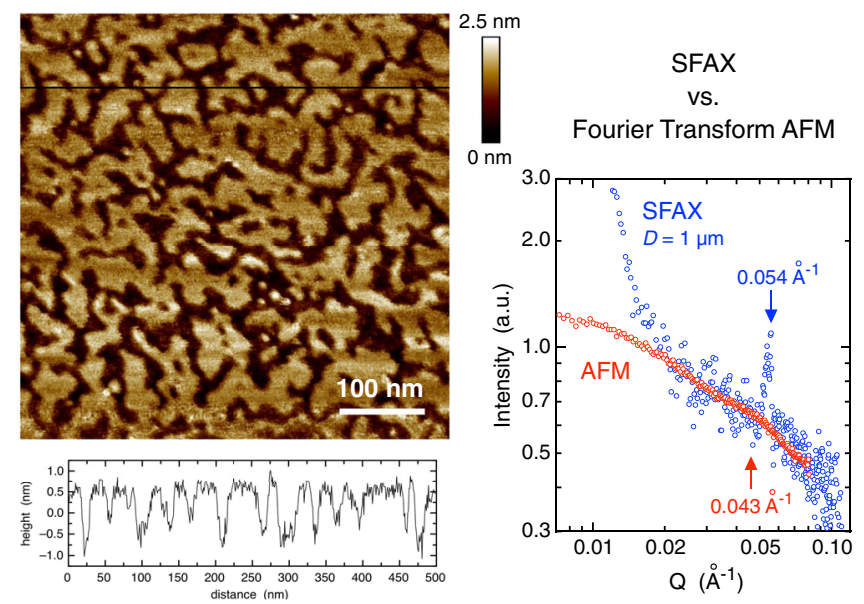

FIG. 4. Left: Peak force mode AFM topographic image of a CTAB-coated mica surface immersed in an aqueous CTAB solution at $\approx 1 / 120 \mathrm{cmc}$ after $24 \mathrm{~h}$ equilibration. The bottom plot shows the height profile along the horizontal black line, revealing bare mica areas surrounded by adsorbed CTAB regions regular in thickness (1.7-1.9 nm). Right: FT of a $2 \mu \mathrm{m} \times 2 \mu \mathrm{m}$ AFM image compared with the $\mathrm{x}$-ray scattering measured at large separations (Fig. 3). At large $Q$, the overall shapes can be superimposed, both revealing diffuse bumps located at similar positions. 
AFM windowing). Details are postponed to a forthcoming article.

The reported accurate synchrotron scattering patterns from within the SFA gap demonstrate that the overall charge neutral CTAB-coated mica substrate is microstructured, with alternating bare and covered patches without long-range order. We also detect similar structures on a single incubated mica sheet by AFM imaging. The variation of the scattering pattern upon confinement strongly suggests counterion release when antagonistic patches happen to face. This provides the ultimate experimental missing clue for explaining the long-range electrostatic attraction between similar, overall charge neutral walls, reported earlier $[1,15]$. This achievement was possible because several improvements of our SFAX setup (to be discussed in more detail elsewhere) were essential since the first attempts performed by others [17,18]. Now it is validated; our accurate SFAX setup should be decisive in many fields of interfacial science (phase transitions under confinement [29,30], ionic liquids, etc.).

C. Contal is gratefully acknowledged for technical help in the AFM experiments. We thank J. Combet and J. Daillant for helpful discussions.

* patrick.kekicheff@ics-cnrs.unistra.fr

[1] R. M. Adar, D. Andelman, and H. Diamant, Electrostatics of patchy surfaces, Adv. Colloid Interface Sci. 247, 198 (2017).

[2] G. Silbert, D. Ben-Yaakov, Y. Dror, S. Perkin, N. Kampf, and J. Klein, Long-Ranged Attraction between Disordered Heterogeneous Surfaces, Phys. Rev. Lett. 109, 168305 (2012).

[3] S. Perkin, N. Kampf, and J. Klein, Long-Range Attraction between Charge-Mosaic Surfaces across Water, Phys. Rev. Lett. 96, 038301 (2006).

[4] R. M. Adar, D. Andelman, and H. Diamant, Electrostatic attraction between overall neutral surfaces, Phys. Rev. E 94, 022803 (2016).

[5] R. Kjellander and S. Marčelja, Inhomogeneous Coulomb fluids with image interactions between planar surfaces. III. Distribution functions, J. Chem. Phys. 88, 7138 (1988).

[6] M. Ghodrat, A. Naji, H. Komaie-Moghaddam, and R. Podgornik, Strong coupling elctrostatics for randomly charged surfaces: antifragility and effective interactions, Soft Matter 11, 3441 (2015).

[7] D. Andelman, Introduction to electrostatics in soft and biological matter, in Soft Condensed Matter Physics in Molecular and Cell Biology, edited by W. C. K. Poon and D. Andelman, Scottish Graduate Series (NATO Adv. Sci. Inst., Scottish Summer School of Physics, 2006), pp. 97-122.

[8] R. Kjellander and S. Marčelja, Perturbation of hydrogen bonding in water near polar surfaces, Chem. Phys. Lett. 120, 393 (1985).

[9] D. J. Bonthuis and R. R. Netz, Beyond the continuum: How molecular solvent structure affects electrostatics and hydrodynamics at solid-electrolyte interfaces, J. Phys. Chem. B 117, 11397 (2013).
[10] M. Kanduč, A. Schlaich, E. Schneck, and R. R. Netz, Hydration repulsion between membranes and polar surfaces: Simulation approaches versus continuum theories, Adv. Colloid Interface Sci. 208, 142 (2014).

[11] A. Schlaich, E. W. Knapp, and R. R. Netz, Water Dielectric Effects in Planar Confinement, Phys. Rev. Lett. 117, 048001 (2016).

[12] L. Herrmann, A. Johner, and P. Kékicheff, Interactions between Charged Lamellae in Aqueous Solution, Phys. Rev. Lett. 113, 268302 (2014).

[13] N. Li, R. K. Thomas, and A. R. Rennie, Effect of pH, surface charge and counter-ions on the adsorption of sodium dodecyl sulfate to the sapphire/solution interface, J. Colloid Interface Sci. 378, 152 (2012).

[14] P. Kékicheff, H. K. Christenson, and B. W. Ninham, Adsorption of cetyltrimethylammonium bromide to mica surfaces below the critical micellar concentration, Colloids Surf. 40, 31 (1989).

[15] P. Kékicheff and O. Spalla, Long-Range Electrostatic Attraction between Similar, Charge-Neutral Walls, Phys. Rev. Lett. 75, 1851 (1995).

[16] O. Spalla and L. Belloni, Long-Range Electrostatic Attraction between Neutral Surfaces, Phys. Rev. Lett. 74, 2515 (1995).

[17] S. H. J. Idziak, C. R. Safinya, R. S. Hill, K. E. Kraiser, M. Ruths, H. E. Warriner, S. Steinberg, K. S. Liang, and J. N. Israelachvili, The X-ray surface forces apparatus: Structure of a thin smectic liquid crystal film under confinement, Science 264, 1915 (1994).

[18] Y. Golan, A. Martin-Herranz, Y. Li, C. R. Safinya, and J. Israelachvili, Direct Observation of a Shear-Induced Orientational Phase Coexistence in a Lyotropic System Using a Modified X-Ray Surface Forces Apparatus, Phys. Rev. Lett. 86, 1263 (2001).

[19] A. R. Khokhlov and I. A. Nyrkova, Compatibility enhancement and microdomain structuring in weakly charged polyelectrolyte mixtures, Macromolecules 25, 1493 (1992).

[20] P. Fontaine, G. Ciatto, N. Aubert, and M. Goldmann, Soft interfaces and resonant investigation on undulator source: A surface X-ray scattering beamline to study organic molecular films at the SOLEIL synchrotron, Sci. Adv. Mater. 6, 2312 (2014).

[21] P. Kékicheff, Direct surface force measurement techniques, in Electrostatic Effects in Soft Matter and Biophysics, edited by C. Holm, P. Kékicheff, and R. Podgornik, NATO Science Series II-Mathematics Physics and Chemistry Vol. 46 (NATO, 2001), pp. 205-281, ISBN: 1-4020-0197-5.

[22] J. N. Israelachvili and G. E. Adams, Measurement of forces between two mica surfaces in aqueous electrolyte solutions in the range 0-100 nm, J. Chem. Soc., Faraday Trans. 1 74, 975 (1978).

[23] J. N. Israelachvili, Thin film studies using multiple-beam interferometry, J. Colloid Interface Sci. 44, 259 (1973).

[24] Note that, at small separations, the freely suspended mica sheets may deform, as seen from the fringes of equal chromatic order in white light [23], and flattening may occur. In any case, the local radius of curvature is measured at every gap width [21].

[25] G. Pletikapić, A. Berquand, T. M. Radić, and V. Svetličić, Quantitative nanomechanical mapping of marine diatom in 
seawater using PeakForce tapping Atomic Force Microscopy, J. Phycol. 48, 174 (2012).

[26] S. M. Loverde, Y.S. Velichko, and M. O. de la Cruz, Competing interactions in two dimensional Coulomb systems: Surface charge heterogeneities in coassembled cationic-anionic incompatible mixtures, J. Chem. Phys. 124, 144702 (2006).

[27] A. Yacoot and L. Koenders, Aspects of scanning force microscope probes and their effects on dimensional measurement, J. Phys. D 41, 103001 (2008).
[28] A. W. Knoll, Nanoscale contact-radius determination by spectral analysis of polymer roughness images, Langmuir 29, 13958 (2013).

[29] K. Binder, J. Horbach, A. Milchev, M. Müller, and R. Vink, Monte Carlo simulations of phase transitions of systems in nanoscopic confinement, Comput. Phys. Commun. 177, 140 (2007).

[30] K. Binder, J. Horbach, R. Vink, and A. de Vigiliis, Confinement effects on phase behavior of soft matter systems, Soft Matter 4, 1555 (2008). 